Pathology and Public Health, then at 62, Queen Annestreet, and at first it was reported as an endothelioma (alveolar sarcoma), but subsequent examinations showed it to be of carcinomatous nature.

The patient improved rapidly after the operation and put on flesh. On May 15th the patient was again seen and was apparently perfectly well, and no abnormal physical signs could be detected anywhere. The scar was very firm. But at the end of July she was again seen and was obviously wasting, with signs of recurrence in the abdomen with free fluid.

Earl's Court, S.W.

\section{NOTE ON AN UNUSUAL CASE OF APPENDICITIS.}

By A. Knyvett Gordon, M.B., B.C. Cantab., MEDICAL SUPERTNTENDENT OF MONSALL FEVER HOSPITAL,

THE following case deserves mention as showing how closely the symptoms and course of enteric fever may be simulated by what was primarily an attack of appendicitis.

The patient, a female, aged 11 years, was admitted into Monsall Hospital on Sept. 10th, 1907, having been certified as suffering from enteric fever; this was apparently the eleventh day of disease. The history was that she had been ill for a year with " nervous debility"; this was, however, apparently subacute rheumatism. On August 31st she was attacked suddenly with headache, diarrhœa, abdominal pain, and vomiting ; these were followed on Sept. 1st by shivering and sweating. As the diarrhoea continued and the abdomen became slightly distended she was notified for removal to hospital.

On admission I found the usual signs of mitral regurgitation and, in addition, there was some bronchitis with diminished air entry at the base of each lung. The tongue was dry and coated in parallel lines. The temperature was $105^{\circ} \mathrm{F}$., the pulse was 140 , and the respirations were 40. There was slight but general abdominal distension. Nothing abnormal could be felt in either iliac fossa or in the region of the bladder. Fairly deep palpation was possible without eliciting any tenderness anywhere. The spleen could not be felt. The lower limit of the liver dulness was half an inch above the costal margin in the right nipple line and the costal margin itself in the anterior axillary line. There was no diarrboa and the stool passed after an enema was dark and partly formed. The Widal reaction gave a negative result. I thought that the patient was probably suffering from a moderately severe attack of enteric fever supervening on subacute rbeumatic endocarditis.

On Sept. 12th there was some abdominal pain which was referred to the umbilical region; this was not accompanied by any symptoms of collapse. The respiratory movement was fair ; the pulse-rate was 110 . The liver dulness was further diminished below by half an inch in the nipple and axillary lines. There was no muscular rigidity and nothing to be felt on deep palpation in the right iliac fossa; there was no tenderness. An ice-bag was applied to the abdomen and the patient was carefully watched The pain passed off almost immediately but recurred at 6.30 P.M. and again at 11.15 P.M. There was no change in the physical signs but the temperature had fallen from $104^{\circ}$ to $101^{\circ}$; the ice-bag was removed. On the 13th there was no more pain but the pulse-rate had risen to 140 and there was some hæmorrhage from the bowel. A definite diagnosis of enteric fever was made. Shortly after mid-day, despite there having been no recurrence of pain, the liver dulness had markedly diminished, though the general abdominal distension had not increased. Perforation of the intestine was suspected and under local anresthesia with $B$ eucaine lactate and adrenalin solution an incision 1 inch in length was made into the peritoneal cavity at the outer edge of the right rectus. As there was no sign of peritonitis whatever and a presenting coil of intestine was normal in appearance the incision was sewn up in layers; there was no free gas in the abdominal cavity. On the 14th the liver dulness had returned to normal. There was more hremorrhage from the bowel. The patient seemed slightly better. On the 16th there was evidently increased toxæmia and there was intermittent abdominal pain. The abdomen was soft and the distension had diminished. The patient died on the 19th, apparently from the toxæmia of enteric fever.

Necropsy.-At the post-mortem examination, made shortly after death, there was no sign of enteric fever whatever and no peritonitis in any situation. The condition of the appendix, however, was peculiar : at its proximal end it was apparently normal ; the tip was adherent to the cæcum lower down and on slitting the latter open an ulcer of the size of half a crown was seen, having an opening into the appendix at its base. The liver was of normal size and on section was. found to be riddled with abscesses of varying size. The patient had obviously suffered from appendicitis in the first instance with pylephlebitis arising by infection from the ulcer in the cæcum. The beart showed the usual signs of chronic mitral disease. The vegetations on the valve were not of recent date. Cultures from the liver gave almost a pure growth of bacillus pyocyaneus.

The interesting points in this case are:-1. The entire absence of any local peritonitis accompanying inflammation of the appendix and of the crecum. In the absence of this it is difficult to see how a diagnosis of appendicitis could bave been made. 2. The entire absence of any hepatic symptoms during life. 3. The closeness with which the toxæmia from the intestinal organisms simulated that of enteric fever. Inasmuch as cultures were not taken during life it is difficult to say whether or not bacillus pyocyanens was the main factor in this case; judging by analogy from similar cases one would suspect that bacillus coli communis was also concerned.

I should add that the abdominal incision was made in con sequence of the difficulty that otherwise existed in determining the absence of perforation. In this case, though the pain was indefinite, the increase in the rapidity of the pulse combined with the loss of liver dulness were taken as an indication for the peritoneal incision, which in itself pro. duced no constitutional disturbance whatever.

Manchester.

\section{NOTE ON A CASE OF RHEUMATIC HYPERPYREXIA} IN A CHILD AGED SIX YEARS. ${ }^{1}$

BY G. H. Lock, M.R.C.S. ExG., L.R.C.P. LoND., D.P.H.

THIs rare and faial complication of acute rheumatism, hitherto, so far as I can discover, unrecorded in so young patient, occurred recently in a girl, aged six years, whom I had attended at birth and since whenever she was ill. Her father had worked in compressed-air caissons for years and always successfully passed the periodical medical examinations to which such workers are subjected. Her mother was young and perfectly healthy. A maternal aunt had suffered from rheumatism; otherwise the family history was free from this disease and good in other respects. The child, with the exception of measles followed by whooping-cough, enjoyed good health during infancy. She grew up healthy, of alert intellect, and engaging disposition and manners. A year ago she passed through an attack of chorea which subsided during six weeks' complete rest in bed, and when she was brought to me in January of this year to report progress she was fit and well with the exception of a rough systolic murmur at the cardiac apex. I could not make out that the heart was appreciably enlarged but the murmur was condncted into the axilla. On Sept. 10th of this year she sickened for left lobar pneumonia which, with a temperature ranging between $102^{\circ}$ and $103^{\circ} \mathrm{F}$. and no uncommon features, terminated by crisis on the fifth day. A day or two after this crisis there was a return of the choreiform movements which at first mild and confined to the left side subsequently became general and severe. On the 20th pain in the left hip was complained of and the temperature rose to $100^{\circ}$. The child was enveloped in cotton-wool and five grains of salicylate of soda in an. alkaline mixture were given every four hours. On the next day (the 21st) other joints were involved, the temperature was $101^{\circ}$, and the case was a typical one of acute rheumatism with chorea. The dose of salicylate of soda was doubled. The following day (the 22nd), at 11 A.M, the temperature was $105^{\circ}$. After tepid sponging the nurse reported a drop to $104 \cdot 5^{\circ}$. Ten grains of salicylate of soda were administered every hour. At 6 P.M. the temperature had risen to $107^{\circ}$. An ice pack was ordered and at 8.30 P.M. the temperature had fallen to $105^{\circ}$. This application of cold was not well borne, alarming cyanosis and embarrassment of respiration followed. The heart distinctly dilated and the sounds were almost inaudible. A drachm of

1 A paper read before the Society for the Study of Disease in Children on Nov. 15th, 1907. 
brandy was given and ordered every hour throughout the night; the salicylate with the addition of three minims of tincture of digitalis was continued. Ice was kept applied to the head and nape of the neck. The next day (the 23rd), at 10 A.M., the temperature was $108^{\circ}$, the pulse was 110, and the respirations were 68 . A graduated bath was prepared, but while waiting for a fresh supply of ice and before immersion the temperature in less than an hour rose to $110^{\circ}$. At this moment death occurred somewhat suddenly. An hour after death the temperature was $109^{\circ}$. These temperatures were taken in the rectum by a trained nurse and verified by me. The last observation was kindly confirmed bs Mr. S. H. Greene of Kensington.

The onset of this period of abnormal temperature was not associated, as far as the chorea would allow me to observe, with any fresh endo- or peri-carditis. The swelling of the joints was not so marked, but it was impossible to obtain anything from the child as to the degree of pain in them. The pulse though feeble was never greatly hurried but the respirations were very frequent (from 60 to 68 ) and noisily panting in character. The most marked feature was the complete suppression of sweating. There was a plentiful crop of sudaminal vesicles from former sweats but the skin was quite dry and pungently hot throughout. There was no sleep during the presence of the hyperpyrexia; on the other hand there was no delirium or mania. The child though dazed asked for and knew her mother to the end and the severe choreic movements ceased only with death. I have searched through many writers for mention of this termination of cerebral rheumatism in childhood with negative results. That it must be extremely rare is evidenced by the remarks of Sir W. S. Church in his article on Rheumatism in Allbutt and Rolleston's "System of Medicine" (1906). In writing of hyperpyrexia he states:

"Its occurrence in early childhood is as yet unknown" and, further, "no age period if we except early childhood appears exempt." In the same volume Dr. W. B. Cheadle, writing under the heading "Rheumatism in Childhood," states that he has never met with a case of hyperpyrexia. The youngest case of which be can find any record is that of a girl, aged 13 years.

Uxbridge-road, $\mathrm{W}$.

\section{attedical Socretties.}

\section{ROYAL SOCIETY OF MEDICINE.}

\section{EPIDEMIOLOGICAL SECTION.}

The Present Methods of Combating the Plague.-The Protec. tion of India from Invasion by Plague.

A MEETING of this section was held on Dec. 2nd, Dr. A. NEWSHOLME, being in the chair

Mr. W. M. HAFFKINE, C.I.E., delivered an address on the Present Methods of Combating the Plague. He said : "The measures suggested for stamping out the plague or for preventing its importation deal (1) with man, (2) with inanimate objects, or (3) with the lower animals. 1. The first category of measures comprises: $(a)$ Discovery and notification of persons attacked with the disease; $(b)$ isolation of the attacked ; $(c)$ certain precautions with regard to the disposal of the dead; $(d)$ segregation of those who have come in contact with the sick or dead; $(e)$ institution of cordons round infected areas ; $(f)$ a less drastic and less thorough plan than the last mentioned-viz., placing in quarantine arrivals from infected places, detaining the sick and suspected, and letting the rest free after a time of observation ; or $(g)$ a still less rigorous measure which is merely to examine travellers, isolate the sick and suspected, and let the others free under a system of surveillance. The following propositions are now more or less generally recognised-viz., that plague is what has been termed, in a general sense, a disease of locality; that it is contracted principally at night; and that the part which man plays as direct agent in its propagation is a more or less subordinate one. This being so, the measures taken with regard to man, even when carried to perfection, can influence the propagation of plague only to the same proportionately limited extent. In the vast majority of cases events have conformed with this conclusion, in that precautions taken against the importation or the spread of plague by man have failed to secure the desired end. It must, however, be stated that these precautions can rarely, if ever, be carried to perfection. Their application is fraught with great difficulties and is often impracticable. This is due to the impossibility of enlisting the self-sacrificing coöperation of private individuals, to the first cases being rarely recognised, to the reluctance of those falling ill to deliver themselves into the hands of strangers and officials, to the measures of prevention hurting trade and numerous private interests, and to the consequent wholesale evasion of prescribed rules. The time, therefore, arrives when the measures directed against man are relaxed or dropped and efforts are chiefly applied to inanimate objects. 2. The list of measures within an area infected comprises in this case destruction or disinfection of houses, furniture, clothing, bedding, carriages, goods, warehouses, grain and other stores, garbage, drains, and streets. Outside the infected area the measures consist in the refusal to admit carts, trains, and ships with goods from infected places, or in the refusal to admit only certain goods, or in mere inspection of trains, carts, and ships, and some procedure by which these, and the goods they convey, as well as the belongings of travellers, are sought to be rendered harmless. All these measures are intended for the avoidance or destruction of plague germs which may possibly exist in the objects concerned. Plague bacilli have, however, been very carefully looked for but have not been so far discovered in the earth, on the walls or floors of houses, or on any inanimate object, unless they were quite obviously and recently soiled by the products of a plague patient. I am not, moreover, aware of an instance in which the propagation of this disease to a distance by means of goods of inanimate nature has been conclusively demonstrated, though linen, clothing, handkerchiefs, and other personal belongings of a sufferer from pneumonic plague, if soiled with his sputum or nasal discharge, must be dangerous for a certain period of time. Further, the microbe of plague is not a resistant organism. From the first it was seen that in ordinary circumstances it was easily killed by disinfectants. Exceptional conditions are required for that microbe to resist and preserve its vitality. In these circumstances the following conclusion might seem justifiable: If inanimate objects are important carriers of plague, disinfection should generally be an effective check to its spread, and on this presumption the above measures were devised. According to numerous observations, however, made by health authorities, a house, its furniture, all the belongings of the inmates, and the inmates themselves may be washed and disinfected repeatedly, and yet cases of plague may occur subsequently to each disinfection if people are allowed to stay in that house. Though general statements to this effect have been made by earnest observers, I am not aware of a precise and convincing demonstration by experiment of a case in which disinfection arrested the development of an epidemic of plague. The above theoretical considerations must be qualified by the following remarks. The expense and the difficulties of destroying or thoroughly disinfecting houses, goods, and other property over a vast area are very great and the agency for properly carrying out such measures is not available or is not forthcoming. The enormous bulk of merchandise conveyed nowadays by rail and ship admits of no process which would answer to elementary requirements of disinfection, and the opposition and hostility of vast numbers of people whose interests are interfered with by these measures can only be estimated when the task is actually undertaken. There is, therefore, little wonder if disinfection fails, as has been seen on so many occasions, to interfere with the progress of the plague. 3 . I come to the measures relating to the lower animals. These are : (a) destruction or keeping away of rats by poisoning, trapping, tar and sulphuric acid mixture, or through the agency of the domestic cat; $(b)$ improvements in towns and villages, with a view of reducing the rat population-viz., structural alterations of dwellings, warehouses, and grain stores, demolition of insanitary buildings, introduction or improvement of conservancy arrangements, prompt disposal of garbage, periodical inspection of stores, paving and draining of streets, and certain other measures; $(c)$ destruction and dispersion of fleas by petroleum or other products; (d) fumigation of houses as a temporary protection against rats and fleas; $(e)$ obligation on ships from infected regions to anchor away from the shore, or $(f)$ provision of mechanical arrangements for preventing the landing of rats along mooring cables and gangways; and $(g)$ fumigation of ships arriving with plague patients or plague rats on 